\title{
Thoracic pedicle subtraction osteotomy in a pediatric patient: a case report
}

\author{
Authors Michael P Silverstein ${ }^{1}$, Selvon F St Clair ${ }^{2}$, Isador H Lieberman ${ }^{3}$ \\ Institutions ${ }^{1}$ College of Medicine, Florida State University, Tallahassee, FL, USA \\ 2 Orthopaedic Institute of Ohio, Lima, OH, USA \\ ${ }^{3}$ Orthopaedic $\&$ Spinal Surgeon, Texas Back Institute, Plano, TX, USA
}

ABSTRACT

Study design: Case report.

Objective: To describe a case of thoracic pedicle subtraction osteotomy (PSO) for congenital kyphosis in a child.

Background information: Although congenital kyphosis is rare, it is a challenging cause of pediatric myelopathy and frank paralysis. Even less common is the use of PSO for the surgical management of focal congenital kyphosis. We present the case of a child with congenital kyphosis that was managed with a pedicle subtraction osteotomy.

Methods: A detailed history and physical examination were performed with careful review of the patient's medical records and x-ray studies. A PSO at T11 was performed along with T9 through L1 instrumented posterolateral fusion.

Case description: A 10-year-old girl was evaluated for walking difficulty and a lump on her back. Physical examination revealed a sharp gibbus kyphosis in the lower thoracic spine with tenderness and bilateral back muscle spasms. The patient displayed difficulty with balance lacking a smooth, regular gait rhythm. Clonus and radiculopathy were not present. Plain x-ray of the thoracolumbar spine revealed hyperkyphosis and failure of anterior wall segmentation between T10 and T11 vertebral bodies. Cobb's angle measured 65 degrees. Due to her symptoms and degree of correction required, we elected to perform a PSO at T11 along with T9 to L1 posterolateral instrumentation fusion. No intraoperative complications occurred. There was a significant improvement in her posture and gait.

Discussion: A thoracic PSO for congenital kyphosis was safely performed with an excellent outcome. To our knowledge, this is the first PSO procedure performed in Uganda. 


\section{INTRODUCTION}

Although congenital kyphosis is rare, if left untreated, it will progress and lead to paraplegia [1-3]. Paralysis occurs most commonly during the pubertal growth spurt. Therefore, to halt the progression of congenital kyphosis, surgery is the primary treatment [4]. Depending on the degree of correction required to re-establish sagittal balance, the current literature supports the use of three procedures: Smith-Peterson osteotomy (SPO) [5], pedicle subtraction osteotomy (PSO) [6-9] and vertebral column resection (VCR) [10]. The SPO offers approximately $10^{\circ}$ of lordotic correction per level and is most often performed in thoracic kyphotic deformities because the cord is not directly manipulated [7]. On average the PSO offers $25^{\circ}$ and $35^{\circ}$ of lordotic correction in the thoracic and lumbar spine, respectively $[6,7,11]$. VCR involves the removal of the entire vertebra and is reserved for patients with severe spinal deformities that require greater than $50 \%$ coronal or sagittal plane correction $[7,10]$.

However, because of the increased risk to the spinal cord and associated procedural morbidity, PSO and VCR in the thoracic spine are sparingly performed. For a successful outcome, spinal deformity correction requires highly skilled surgical teams, careful surgical planning, and the judicious use of some form of spinal cord monitoring to avoid spinal cord-related morbidity.
This report presents a child with focal congenital kyphosis and gait abnormality. To halt the natural history of the disease and prevent further neurological dysfunction, PSO, and posterolateral fusion was performed. The case presentation, radiological findings, surgical technique, and postoperative outcomes are presented.

\section{METHODS}

A detailed history and physical examination were performed along with careful review of the patient's medical records. Radiographic studies were also reviewed. Pedicle subtraction osteotomy at T11 was performed along with T9 through Ll instrumented posterolateral fusion.

\section{REPORT OF A CASE}

A 10-year-old girl with pain and gait difficulty presented to the spine ward at Mulago Hospital in Uganda. She was evaluated by a team of visiting surgeons. During physical examination, a sharp gibbous-type kyphotic curvature was noted. Tenderness and bilateral muscle spasm were noted during examination. Neurological examination revealed difficulty with balance and irregular gait rhythm.
Fig 1a-b Preoperative AP (a) and lateral (b) plain x-ray film of the thoracic spine showing hyperkyphosis and failure of anterior wall segmentation between the T10 and T11 vertebral bodies.

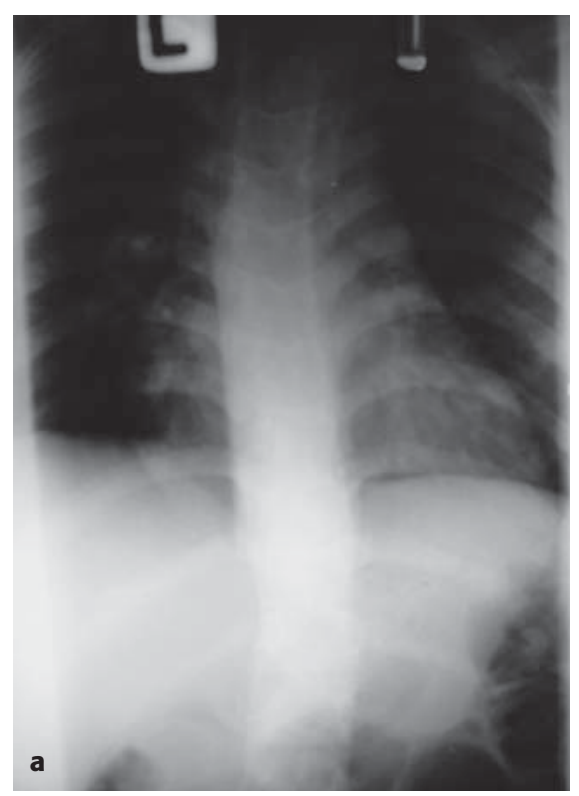

Fig 2 Intraoperative digital photograph of the "eggshelled" pedicles of T11.

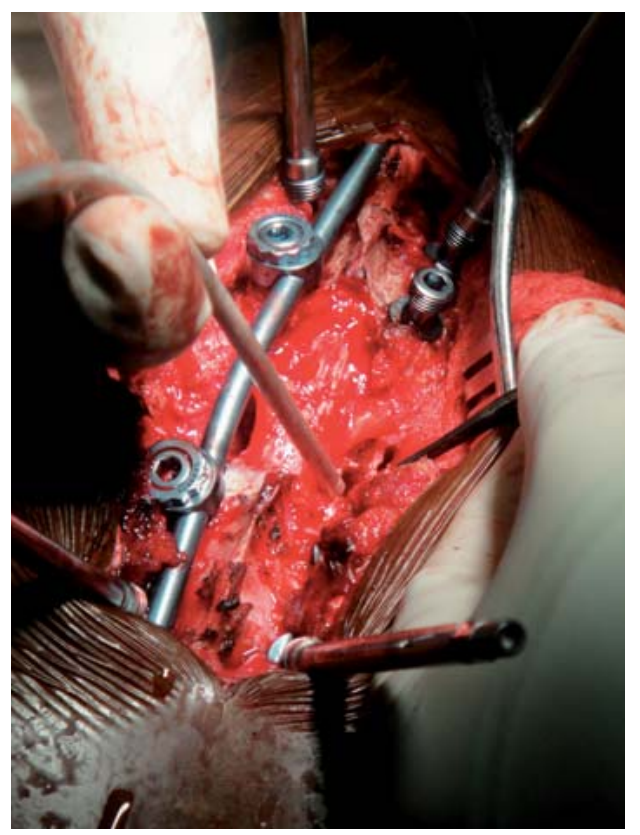


Clonus and radiculopathy were not present. Plain x-ray of the thoracolumbar spine revealed hyperkyphosis and failure of anterior wall segmentation between T10 and T11 vertebral bodies (Fig 1). Segmental sagittal Cobb angle measured $65^{\circ}$. Magnetic resonance imaging was not available. Due to the patient's symptoms and the degree of correction required, we recommended to the caregivers (she was an orphan) that she undergoes a PSO at Tll along with T9 to L1 posterolateral instrumentation.

The surgical equipment and supplies in Uganda are rudimentary and limited. Following the induction of general anesthesia, the patient was positioned prone on a Hall Relton spine frame. Spinal cord monitoring and cell saver autotransfusion, routinely used in developed countries, were not available. Since intraoperative imaging was not available, the incision was carefully delineated by identification of anatomical landmarks, namely rib counting and Macnab's triangle (intersection of 12th rib and transverse process of L1). After a midline incision, thorough subperiosteal dissection was carried out to expose the posterior elements of T9 through Ll. To minimize bleeding, epinephrine-soaked gauzes were used to pack the dissected paraspinal muscles. Following exposure, posterior column ligament and facet releases were performed. After subperiosteal dissection, the medial aspect of the T11 rib was removed. Next, the dissection was extended around the lateral aspect of the cranial and caudal pedicles and T11 vertebral body, while protecting the soft tissues with malleable retractors.
Prior to performing osteotomy, pedicle screws were placed at T9, T10, T12, and L1 with a temporary stabilizing rod on the right to prevent subluxation and potential cord injury. A wide central laminectomy was then performed at Tll along with partial resection of the spinous processes and laminotomies of adjacent levels to provide adequate room for the redundant dura once the osteotomy is closed. Epidural and osseous bleeding were controlled with gelfoam (Pfizer, New York, NY) and bone wax, respectively. The pedicles of T11 were circumferentially exposed to allow for transpedicular "eggshell" decancellization (Fig 2). This was followed with T10/T11 discectomy, lateral wall osteotomy, pedicle resection, and collapse of posterior cortex. Lordotic correction was applied over the temporary rod, followed by placement of the permanent left rod. The temporary rod was exchanged for a permanent right rod. Final compression with visible correction of the vertebral column occurred. Bone graft was then placed in the posterolateral gutters along T9 through L1. The exposed dura was covered with a piece of gelfoam (Pfizer, New York, NY). There were no complications related to the placement of hardware and the estimated blood loss was $450 \mathrm{cc}$.

At a most recent follow-up, the patient was neurologically intact. There was a significant improvement in her posture (Fig 3). Postoperative plain x-rays also show a significant improvement in a sagittal alignment with a Cobb's angle measurement of $18^{\circ}$ (Fig 4).
Fig 3 Clinical photograph of the patient on postoperative day 4 showing an upright posture.

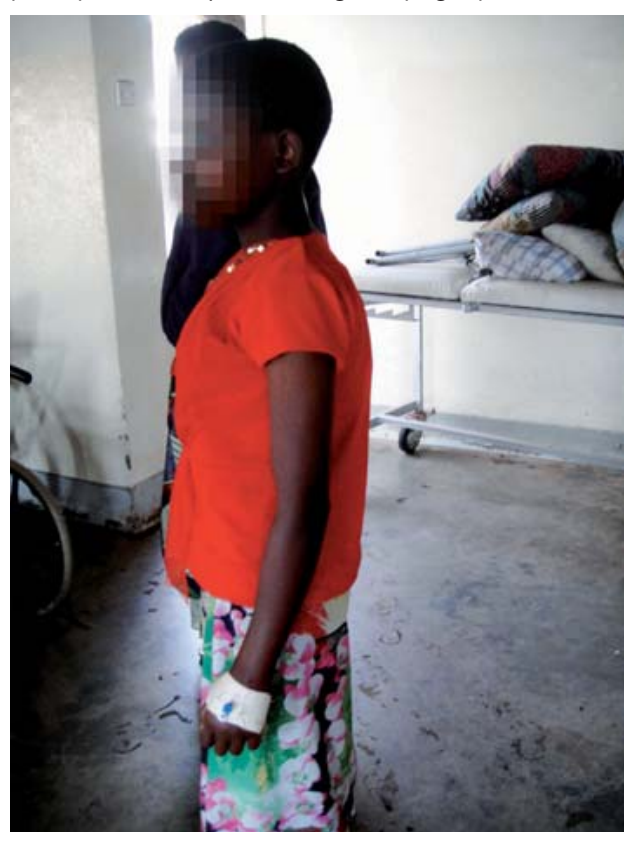

Fig 4a-b Postoperative AP (a) and lateral (b) plain x-ray films of the thoracic spine.
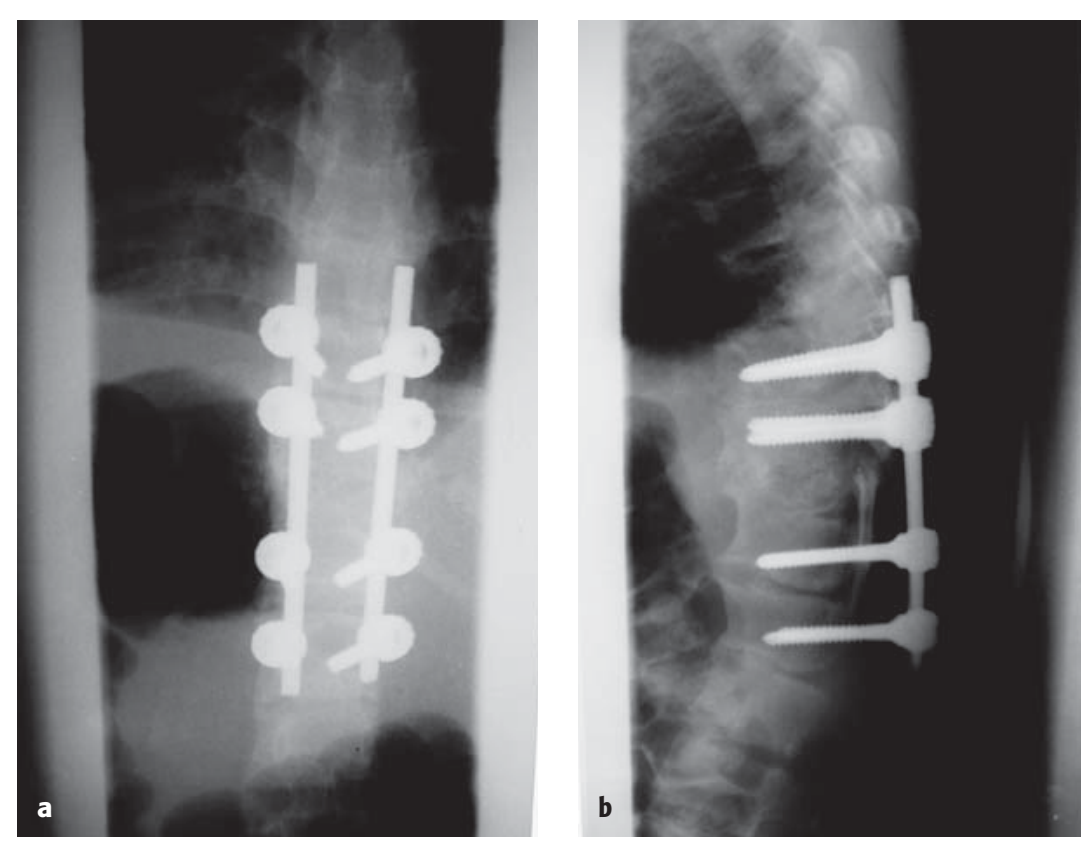


\section{DISCUSSION}

Congenital kyphosis is less common than congenital scoliosis. In the United States, it is the most frequent cause of paraplegia due to spinal deformity $[1,2,12]$. Since bracing has been shown to be ineffective, the primary treatment for congenital kyphosis is surgery [1-3]. The main goal of surgery is to prevent progression and possible neurological complications caused by spinal cord compression. Particularly important for successful surgical outcome is the choice of surgical procedure for the specific deformity correction. There is more than one surgical option available and the one selected is often dictated by the experience of the surgeon, the age of the patient, the magnitude of the deformity, and the patient's neurological symptoms.

The differences in curvature and the region of the spine that is affected are part of the preoperative evaluation in determining the most appropriate surgical technique. The Smith-Peterson extension osteotomy technique has been commonly used for reconstruction of sagittal imbalance in patients with deformity above the thoracolumbar junction $[5,7,13]$. Total correction over several spinal segments from $20^{\circ}$ to $40^{\circ}$ is achievable $[5,7,13]$. Caveats of the Smith-Peterson technique are the need for multiple osteotomies and the occasional use of anterior osteotomy for effective posterior compression. Additionally, this approach lengthens the spinal column and has the potential for neurological injury from stretch of the spinal cord [14]. Modified from the initial technique described by Simmons [9], PSO is gaining popularity among deformity surgeons $[4,6,8,11,15-17]$. Pedicle subtraction osteotomy is typically used for deformities below cord levels with reported corrections of $35^{\circ}$ to $40^{\circ}$ in the lumbar spine. A major advantage of PSO is inherent in the technique, which creates a wedge with the fulcrum at the anterior column, thereby allowing for three column contact upon closure [6]. This creates a better environment for fusion. Additionally, the spinal column is effectively shortened which minimizes the risk of compression and stretch injury to the cord.

The performance of PSO procedures for thoracic kyphosis in children is far less frequently reported than for the lumbar spine [18]. Furthermore, to our knowledge, the performance of this procedure in a developing country has not been documented before. In part, this is due to the lack of the necessary surgical equipment, medical supplies, and skilled personnel needed to consistently have safe outcomes. In our case, there were no intraoperative imaging devices available to assist with localization. Advanced imagings, such as MRI and CT 3-D reconstruction, were not available to assess for epidural and intradural pathology and assist with preoperative planning. Although we do not advocate the widespread use of PSO procedures under these conditions, this case report demonstrates that it can be done safely with an excellent outcome. The alternative for this patient was nonsurgical management which has been shown to have a poor outcome manifested by progressive myelopathy [1-3].

This case report describes the successful execution of a PSO procedure on a 10-year-old girl in Uganda with myelopathic symptoms caused by congenital kyphosis. To our knowledge this is one of a few reports of thoracic PSO in a patient younger than 10 years and certainly the first report of such a procedure performed in a developing country.

\section{REFERENCES}

1. Marks DS, Qaimkhani SA (2009) The natural history of congenital scoliosis and kyphosis. Spine (Phila Pa 1976); 34(17):1751-1755.

2. McMaster MJ, Singh H (1999) Natural history of congenital kyphosis and kyphoscoliosis: a study of one hundred and twelve patients. J Bone Joint Surg Am; 81(10):1367-1383.

3. Winter RB, Moe JH, Wang JF (1973) Congenital kyphosis: its natural history and treatment as observed in a study of one hundred and thirty patients. $J$ Bone Joint Surg Am; 55(2):223-256.

4. Noordeen MH, Garrido E, Tucker SK, et al (2009) The surgical treatment of congenital kyphosis. Spine (Phila Pa 1976); 34(17):1808185.

5. Smith-Petersen MN, Larson CB, Aufranc OE (1969) Osteotomy of the spine for correction of flexion deformity in rheumatoid arthritis. Clin Orthop Relat Res; 66:6-9.

6. Bridwell KH, Lewis SJ, Rinella A (2004) Pedicle subtraction osteotomy for the treatment of fixed sagittal imbalance: surgical technique. Bone Joint Surg Am; 86-A Suppl 1:44-50.

7. Gill JB, Levin A, Burd T, et al (2008) Corrective osteotomies in spine surgery. J Bone Joint Surg Am; 90(11):2509-2520.

8. Ikenaga M, Shikata J, Takemoto $M$, et al (2007) Clinical outcomes and complications after pedicle subtraction osteotomy for correction of thoracolumbar kyphosis. J Neurosurg Spine; 6(4):330-336.

9. Simmons EH (1972) The surgical correction of flexion deformity of the cervical spine in ankylosing spondylitis. Clin Orthop Relat Res; 86:132-143. 


\section{EDITORIAL PERSPECTIVE}

The authors deserve praise for their wonderful and seemingly successful work. Our commentator, who is no stranger to working in so-called third world countries provided a differentiated perspective on the technical issues surrounding kyphocorrection surgery in the thoracolumbar spine. This case report points out two unresolved issues.

First, there are several ways to perform corrective osteotomies in thoracolumbar deformities. So far there are no clear advantages of any technique over others, all of them have some advantages and each features a number of drawbacks. All techniques have in common the need of a surgeon well trained and familiar with the technique to apply them as well and as safely as possible. Silverstein and colleagues note that all these techniques are resource intense and require a suitable infrastructure. This resource-rich environment was not really given in the example described by the authors as they performed a sophisticated surgery very nicely in a complex environment as an act of humanitarian help.

10. Lenke LG, O’Leary PT, Bridwell KH, et al (2009) Posterior vertebral column resection for severe pediatric deformity: minimum two-year follow-up of thirty-five consecutive patients. Spine (Phila Pa 1976); 34(20):22132221.

11. Kim YJ, Bridwell KH, Lenke LG, et al (2007) Results of lumbar pedicle subtraction osteotomies for fixed sagittal imbalance: a minimum 5-year follow-up study. Spine (Phila Pa 1976); 32(20):2189-2197.

12. Guille JT, Forlin E, Bowen JR, et al (1993) Congenital kyphosis. Orthop Rev; 22(2):235239.

13. Bridwell KH (2006) Decision making regarding Smith-Petersen vs. pedicle subtraction osteotomy vs. vertebral column resection for spinal deformity. Spine (Phila Pa 1976); 31 (19 Suppl):S171-178.

14. La Marca F, Brumblay H (2008) Smith-Petersen osteotomy in thoracolumbar deformity surgery. Neurosurgery; 63(3 Suppl):163-170.

15. Gertzbein SD, Harris MB (1992) Wedge osteotomy for the correction of post-traumatic kyphosis: a new technique and a report of three cases. Spine (Phila Pa 1976); 17(3):374-379.

16. Murrey DB, Brigham CD, Kiebzak GM, et al (2002) Transpedicular decompression and pedicle subtraction osteotomy (eggshell procedure): a retrospective review of 59 patients. Spine (Phila Pa 1976); 27(21):2338-2345.

17. O'Shaughnessy BA, Kuklo TR, Hsieh PC, et al (2009) Thoracic pedicle subtraction osteotomy for fixed sagittal spinal deformity. Spine (Phila Pa 1976); 34(26):2893-2899.

18. Mummaneni PV, Dhall SS, Ondra SL, et al (2008) Pedicle subtraction osteotomy. Neurosurgery; 63(3 Suppl):171-176.
This raises the second concern: where should such a case take place if a desirable infrastructure is not really present? Should the case- as was done-be treated locally by a group of outstanding surgeon volunteers who make the best use of what's available and accept a number of compromises (ie, blood supply, antisepsis, imaging, neuromonitoring, ICU facilities)? Or should an attempt be made to bring the patient to a more well-equipped facility with adequate resources, perhaps even in another country? If option two is chosen, who would pay for such an effort?

In case of a severe complication, such as perioperative paralysis, either option would however result in unforeseeable consequences without a suitably developed social safety network. However, not doing anything in a young patient with myelopathy in presence of a treatable deformity does not seem to be an ethically acceptable answer either. As our world seems to progressively shrink through travel exposure, better communications, and more consistent education we will undoubtedly face similar dilemmas more commonly. As much as we admire successful acts of individual civility, such as was done here, there appears to be no ready answers to bringing surgical solutions in spine care to all those in need around the globe.

With this editorial perspective, EBSJ invites the thoughts of its readership on this contentious issue and asks for your comments for possible future publication. What would you recommend doing for an adolescent with progressive myelopathy in case of thoracic hyperkyphosis? Treat as was done here, locally, with some improvisation, a lot of experience and a lot of heart? Find suitable receiving country with necessary infrastructure and with a return home provision for the patient mindful of diverse scenarios? Observe patient nonoperatively? Other options? Please send your thoughts to EBSJ at ebsj@specri.com. 


\section{COMMENTARY}

Richard J Bransford, $M D$

Dept of Orthopaedics and Sports Medicine

Harborview Medical Center

Seattle, WA, USA

Silverstein and colleagues very nicely illustrate the case of a 10-year-old girl with a $65^{\circ}$ kyphosis secondary to a failure of segmentation of T10-T11who presented with myelopathy. The goal in this case was to decompress the neural elements and correct the deformity. The typical tools available for sagittal imbalance include the following:

- Ponte or Smith-Peterson osteotomies that can usually achieve about $10^{\circ}$ of correction per level [1,2].

- Pedicle subtraction osteotomy (PSO) that classically has been able to achieve about $35^{\circ}$ of correction per level [3].

- Vertebral column resection (VCR) that typically has been able to achieve about $50^{\circ}$ of correction per level [4].

Based on these standard options, the standard of care to correct a $65^{\circ}$ kyphosis should have been a VCR with the hope of correcting the deformity to $\sim 15^{\circ}$. There are a couple of issues that are a little unique in this case. First, there is a failure of segmentation between T10 and T11, therefore trying to do a VCR at T11 may be difficult without also sacrificing some or all of T10. Second, a $V C R$ typically requires something to replace the vertebral body, such as a cage or allograft, which may not have been available in Uganda where resources would most likely be limited.

The authors were able to correct the kyphosis from $65^{\circ}$ to $18^{\circ}$ a $47^{\circ}$ correction-which is generally more than is normally expected with PSO. PSOs are usually advocated for use in the lumbar spine below the conus, and are typically believed to be dangerous in the thoracic spine secondary to the risk of spinal cord injury. There is a shortening of the posterior and middle columns which may cause kinking of the neural elements and redundancy of the dura. There certainly is a large body of literature advocating the use of PSOs in the lumbar spine, as has been pointed out by the authors, but minimal literature on its use in the thoracic spine. A recent article by O'Shaughnessy et al [5] looking at 25 thoracic PSOs showed a mean correction of $16.3^{\circ}+1-9.6^{\circ}$. Silverstein et al in this case report were able to achieve an impressive $47^{\circ}$ correction through their PSO at T11. If I were faced with this case, I would have been inclined to do a 2-level VCR to try to correct this deformity. I would not have expected that I could achieve a physiological correction of a $65^{\circ}$ curve with PSO only. I propose that the procedure done here is outside "the standard of care" because the literature would suggest that PSO is "not enough" to correct this severe deformity and generally would have come up short of the goal of achieving sagittal balance. I think the authors recognize this as well and clearly state that they "do not advocate the widespread use of PSO procedures" under the conditions but their only other alternative for this patient was nonsurgical management. Certainly as well, one cannot always abide by "the standard of care" in developing countries and often this has to be redefined. The proof is in the pudding, and the surgeons were able to achieve far beyond what was expected from PSO and obtain an acceptable correction as well as maintaining and improving the patient's neurological status. Financially, they were also able to do this in a much less expensive way as no interbody cage or allograft was needed as would have been required with VCR.

Well done!

1. Ponte A, Vero B, Siccardi GL (1984) Surgical treatment of Scheuermann's hyperkyphosis. In: Winter RB, ed. Progress in Spinal Pathology: Kyphosis. Bologna: Aulo Gaggi; 75-80.

2. Smith-Petersen MN, Larson CB, Aufranc OE (1945) Osteotomy of the spine for correction of flexion deformity in rheumatoid arthritis. Clin Orthop Relat Res; 66:6-9.

3. Thomasen E (1985) Vertebral osteotomy for correction of kyphosis in ankylosing spondylitis. Clin Orthop Relat Res; 194:142-152.

4. Boachie-Adjei O, Bradford DS (1991) Vertebral column resection and arthrodesis for complex spinal deformities. J Spinal Disord; 4(2):193-202.

5. O'Shaughnessy BA, Kuklo TR, Hsieh PC, et al (2009) Thoracic pedicle subtraction osteotomy for fixed sagittal spinal deformity. Spine (Phila Pa 1976); 15;34(26):2893-2899. 